\title{
Mehar Methods for Fuzzy Optimal Solution and Sensitivity Analysis of Fuzzy Linear Programming with Symmetric Trapezoidal Fuzzy Numbers
}

\author{
Sukhpreet Kaur Sidhu, ${ }^{1}$ Amit Kumar, ${ }^{1}$ and S. S. Appadoo ${ }^{2}$ \\ ${ }^{1}$ School of Mathematics and Computer Applications, Thapar University, Patiala, Punjab 147004, India \\ ${ }^{2}$ Department of Supply Chain Management, University of Manitoba, Winnipeg, MB, Canada R3T2N2 \\ Correspondence should be addressed to S. S. Appadoo; appadoo@cc.umanitoba.ca
}

Received 3 December 2013; Revised 11 February 2014; Accepted 19 March 2014; Published 22 April 2014

Academic Editor: Jun Jiang

Copyright (c) 2014 Sukhpreet Kaur Sidhu et al. This is an open access article distributed under the Creative Commons Attribution License, which permits unrestricted use, distribution, and reproduction in any medium, provided the original work is properly cited.

\begin{abstract}
The drawbacks of the existing methods to obtain the fuzzy optimal solution of such linear programming problems, in which coefficients of the constraints are represented by real numbers and all the other parameters as well as variables are represented by symmetric trapezoidal fuzzy numbers, are pointed out, and to resolve these drawbacks, a new method (named as Mehar method) is proposed for the same linear programming problems. Also, with the help of proposed Mehar method, a new method, much easy as compared to the existing methods, is proposed to deal with the sensitivity analysis of the same type of linear programming problems.
\end{abstract}

\section{Introduction}

Linear programming is one of the most successively applied operation research techniques. Real world situations are represented by using any linear programming model which involves a lot of parameters, whose values are assigned by experts. However, both experts and decision maker frequently do not precisely know the value of those parameters. Therefore, it is useful to consider the knowledge of experts about the parameters as fuzzy data [1]. In this section, the work which has been done in recent years for such linear programming problems, in which the imprecise parameters are represented by symmetric triangular/trapezoidal fuzzy numbers, is reviewed.

Ganesan and Veeramani [2] proposed new product of symmetric trapezoidal fuzzy numbers and proposed a method to find the fuzzy optimal solution of such singleobjective linear programming problems in which coefficients of the constraints are represented by real numbers and all the other parameters as well as variables are represented by symmetric trapezoidal fuzzy numbers.
Nasseri and Mahdavi-Amiri [3] proposed the dual for the existing symmetric fuzzy linear programming problems [2] and proposed some duality results. Nasseri et al. [4] proved weak and strong duality theorems for the existing symmetric fuzzy linear programming problems [2].

Ebrahimnejad et al. [5] extended the existing symmetric fuzzy linear programming problem [2] to bounded symmetric fuzzy linear programming problem and proposed a method for solving these problems.

Ebrahimnejad [6] pointed out that existing method [2] can be used only if it is easily possible to find a basic feasible solution of primal problem. However, if this condition is not satisfied then existing method [2] cannot be used. To overcome these limitations, Ebrahimnejad [6] proposed a primal dual simplex algorithm for solving these problems.

Ebrahimnejad and Nasseri [7] proposed a duality approach to solve linear programmes with trapezoidal fuzzy numbers. Kumar and Kaur [8] proposed an alternative method for solving existing symmetric fuzzy linear programming problems [2].

Ebrahimnejad [9] proved that if fuzzy linear programming problem considered by Ganesan and Veeramani [2] had 
TABLE 1: Optimal table for the existing problem $\left(\mathrm{P}_{2}\right)$ [11, Example 1, pp. 178] obtained by using the existing method [11].

\begin{tabular}{|c|c|c|c|c|c|c|c|}
\hline Basis & $\tilde{x}_{1}$ & $\tilde{x}_{2}$ & $\tilde{x}_{3}$ & $\tilde{x}_{4}$ & $\tilde{x}_{5}$ & $\tilde{x}_{6}$ & RHS \\
\hline$\widetilde{z}$ & $\widetilde{0}$ & $\tilde{0}$ & $\left(\frac{147}{20}, \frac{229}{20}, \frac{22}{5}, \frac{22}{5}\right)$ & $\widetilde{0}$ & $\left(\frac{37}{20}, \frac{51}{20}, \frac{4}{5}, \frac{4}{5}\right)$ & $\left(\frac{12}{5}, \frac{14}{5}, \frac{3}{5}, \frac{3}{5}\right)$ & \\
\hline$\tilde{x}_{4}$ & 0 & 0 & $-\frac{7}{5}$ & 1 & $-\frac{1}{5}$ & $-\frac{3}{5}$ & $\left(-4,28, \frac{48}{5}, \frac{48}{5}\right)$ \\
\hline$\tilde{x}_{1}$ & 1 & 0 & $\frac{3}{4}$ & 0 & $-\frac{1}{4}$ & 0 & $(15,20,2,2)$ \\
\hline$\tilde{x}_{2}$ & 0 & 1 & $-\frac{3}{10}$ & 0 & $\frac{1}{10}$ & $-\frac{1}{5}$ & $\left(5,13, \frac{9}{5}, \frac{9}{5}\right)$ \\
\hline
\end{tabular}

a fuzzy feasible solution, then it also has fuzzy basic feasible solution, and if it has an optimal fuzzy solution, then it has an optimal fuzzy basic solution.

Ebrahimnejad and Verdegay [10] proposed a new method to deal with the sensitivity analysis of that kind of fuzzy linear programming problems in which the elements of the coefficient matrix of the constraints are represented by real numbers and remaining parameters are represented by symmetric trapezoidal fuzzy numbers.

Kheirfam and Verdegay [11] proposed an approach, based on dual simplex method, for existing symmetric fuzzy linear programming problems [2] and also proposed a method to deal with the sensitivity analysis of these problems.

In this paper, drawbacks of existing methods [2, 4-7, 911] are pointed out and alternative methods (named as Mehar methods), which are much easy to apply as compared to existing methods $[2,4-7,9-11]$, are proposed. The proposed Mehar methods are illustrated with the help of existing numerical problems.

\section{Drawbacks of Existing Methods}

Ganesan and Veeramani [2] proposed a product of symmetric trapezoidal fuzzy numbers and proposed a method to find fuzzy optimal solution of symmetric fuzzy linear programming problem $\left(\mathrm{P}_{1}\right)$. Since then the different methods [47, 9-11] have been proposed for the same linear programming problems.

In this section, drawbacks of all these existing methods $[2,4-7,9-11]$ are pointed out.

$\operatorname{Problem}\left(\mathrm{P}_{1}\right)$.

One has

$$
\begin{aligned}
& \operatorname{maximize}\left[\tilde{z} \approx \sum_{j=1}^{n} \widetilde{c}_{j} \otimes_{G} \widetilde{x}_{j}\right] \\
& \text { subject to } \sum_{j=1}^{n} a_{i j} \widetilde{x}_{j} \approx \widetilde{b}_{j}, \quad i=1,2, \ldots, m, \\
& \tilde{x}_{j} \geq \widetilde{0}, \quad j=1,2, \ldots, n,
\end{aligned}
$$

where $\widetilde{c}_{j}, \tilde{x}_{j}$, and $\widetilde{b}_{j}$ are symmetric trapezoidal fuzzy numbers and $a_{i j}$ are real numbers.
In the crisp environment, an alternative optimal solution of a crisp linear programming problem will not exist if the relative profit corresponding to none of the basic variables is zero in the optimal table [12]. On the same direction, in all the existing methods $[2,4-7,9-11]$, it is assumed that if the rank of relative fuzzy profit corresponding to none of the fuzzy basic variables is zero in the optimal table, then alternative fuzzy optimal solution will not exist.

for example, Kheirfam and Verdegay [11] solved the problem $\left(\mathrm{P}_{2}\right)$ to illustrate their proposed method and concluded that Table 1 is the optimal table of this problem. Since, in Table 1, the rank of the fuzzy relative profit corresponding to none of the fuzzy basic variables is zero, according to the existing methods [2, 4-7, 9-11] there will not exist any alternative fuzzy optimal solution for this fuzzy linear programming problem.

However, it can be easily verified that all the symmetric trapezoidal fuzzy numbers $\widetilde{x}_{1}, \widetilde{x}_{2}$, and $\widetilde{x}_{3}$ such that $\mathfrak{R}\left(\widetilde{x}_{1}\right)=$ $35 / 2, \mathfrak{R}\left(\tilde{x}_{2}\right)=9$, and $\mathfrak{R}\left(\tilde{x}_{3}\right)=0$ will be the fuzzy optimal solutions of the problem $\left(\mathrm{P}_{2}\right)$; for example, it can be easily verified that the following four feasible solutions are also fuzzy optimal solutions of problem $\left(\mathrm{P}_{2}\right)$.

$$
\begin{aligned}
& \text { (1) } \tilde{x}_{1}=(10,25,3,3), \tilde{x}_{2}=(8,10,2,2), \tilde{x}_{3}=(-1,1,3,3) . \\
& \text { (2) } \tilde{x}_{1}=(17,18,4,4), \tilde{x}_{2}=(9,9,3,3), \tilde{x}_{3}=(-3,3,4,4) . \\
& \text { (3) } \tilde{x}_{1}=(12,23,3,3), \tilde{x}_{2}=(6,12,4,4), \tilde{x}_{3}=(-2,2,3,3) . \\
& \text { (4) } \tilde{x}_{1}=(16,19,2,2), \tilde{x}_{2}=(7,11,8,8), \tilde{x}_{3}=(-6,6,5,5) .
\end{aligned}
$$

Problem $\left(P_{2}\right)$ [11, Example 1, pp. 178].

One has

$$
\begin{aligned}
& \operatorname{maximize}\left[\widetilde{z} \approx-(13,15,2,2) \tilde{x}_{1}\right. \\
& \left.-(12,14,3,3) \tilde{x}_{2}-(15,17,2,2) \tilde{x}_{3}\right] \\
& \text { subject to } 2 \tilde{x}_{1}+3 \tilde{x}_{2}+2 \tilde{x}_{3} \geq(45,55,6,6) \text {, } \\
& 4 \tilde{x}_{1}+3 \tilde{x}_{3} \succeq(60,80,8,8), \\
& 2 \tilde{x}_{1}+5 \tilde{x}_{2} \geq(65,95,5,5), \\
& \tilde{x}_{1} \succeq \tilde{0}, \quad \tilde{x}_{2} \succeq \tilde{0}, \quad \tilde{x}_{3} \succeq \tilde{0} \text {. }
\end{aligned}
$$




\section{Proposed Mehar Method for Finding Fuzzy Optimal Solution}

In this section, to resolve the drawbacks of the existing methods [2, 4-7, 9-11], pointed out in Section 2, an alternative method (named as Mehar method) for finding the fuzzy optimal solution of problem $\left(\mathrm{P}_{1}\right)$ is proposed.

The steps of the proposed Mehar method are as follows.

Step 1. Using Definition 9 of the appendix, the problem $\left(\mathrm{P}_{1}\right)$ can be converted in to problem $\left(\mathrm{P}_{3}\right)$ :

$$
\begin{aligned}
\operatorname{maximize} & {\left[\mathfrak{R}(\widetilde{z})=\mathfrak{R}\left(\sum_{j=1}^{n} \widetilde{c}_{j} \otimes_{G} \tilde{x}_{j}\right)\right] } \\
\text { subject to } & \Re\left(\sum_{j=1}^{n} a_{i j} \tilde{x}_{j}\right)=\mathfrak{R}\left(\widetilde{b}_{i}\right), \quad i=1,2, \ldots, m, \\
& \mathfrak{R}\left(\tilde{x}_{j}\right) \geq \mathfrak{R}(\widetilde{0}), \quad j=1,2, \ldots, n .
\end{aligned}
$$

Step 2. Using the properties $\mathfrak{R}\left(\sum_{j=1}^{n} \widetilde{c}_{j} \otimes_{G} \tilde{x}_{j}\right)=$ $\sum_{j=1}^{n} \mathfrak{R}\left(\widetilde{c}_{j} \otimes_{G} \tilde{x}_{j}\right)=\sum_{j=1}^{n} \mathfrak{R}\left(\widetilde{c}_{j}\right) \mathfrak{R}\left(\tilde{x}_{j}\right)$ and $\mathfrak{R}\left(a_{i j} \widetilde{x}_{j}\right)=a_{i j} \mathfrak{R}\left(\tilde{x}_{j}\right)$ the problem $\left(\mathrm{P}_{3}\right)$ can be converted into problem $\left(\mathrm{P}_{4}\right)$ :

$$
\begin{aligned}
& \text { maximize }\left[\mathfrak{R}(\widetilde{z})=\sum_{j=1}^{n} \mathfrak{R}\left(\widetilde{c}_{j}\right) \mathfrak{R}\left(\tilde{x}_{j}\right)\right] \\
& \text { subject to } \sum_{j=1}^{n} a_{i j} \mathfrak{R}\left(\tilde{x}_{j}\right)=\mathfrak{R}\left(\widetilde{b}_{i}\right), \quad i=1,2, \ldots, m, \\
& \qquad \mathfrak{R}\left(\tilde{x}_{j}\right) \geq \mathfrak{R}(\widetilde{0}), \quad j=1,2, \ldots, n .
\end{aligned}
$$

Step 3. Since $\Re(\widetilde{A})$ is a real number, assuming $\Re\left(\widetilde{c}_{j}\right)=c_{j}$, $\mathfrak{R}\left(\widetilde{b}_{i}\right)=b_{i}, \mathfrak{R}(\widetilde{z})=z$, and $\mathfrak{R}\left(\widetilde{x}_{j}\right)=x_{j}$ and putting $\mathfrak{R}(\widetilde{0})=0$, the problem $\left(\mathrm{P}_{4}\right)$ can be converted into problem $\left(\mathrm{P}_{5}\right)$ :

$$
\begin{array}{ll}
\operatorname{maximize} & {\left[z=\sum_{j=1}^{n} c_{j} x_{j}\right]} \\
\text { subject to } & \sum_{j=1}^{n} a_{i j} x_{j}=b_{i}, \quad i=1,2, \ldots, m, \\
& x_{j} \geq 0, \quad j=1,2, \ldots, n .
\end{array}
$$

Step 4. Use an appropriate existing method [12] to find the optimal solution of the problem $\left(\mathrm{P}_{5}\right)$.

Step 5. Since there exist infinite symmetric trapezoidal fuzzy numbers having the same rank. So, if $x_{1}=a_{1}, x_{2}=$ $a_{2}, \ldots, x_{n}=a_{n}$ is an optimal solution of the problem $\left(\mathrm{P}_{5}\right)$ then all the symmetric trapezoidal fuzzy numbers $\tilde{x}_{1}, \tilde{x}_{2}, \ldots, \tilde{x}_{n}$ such that $\mathfrak{R}\left(\tilde{x}_{1}\right)=a_{1}, \mathfrak{R}\left(\tilde{x}_{2}\right)=a_{2}, \ldots, \mathfrak{R}\left(\tilde{x}_{n}\right)=$ $a_{n}$ will be the fuzzy optimal solution of the problem $\left(\mathrm{P}_{1}\right)$.

\section{Proposed Mehar Method to Deal with the Sensitivity Analysis}

Kheirfam and Verdegay [11] as well as Ebrahimnejad and Verdegay [10] proposed a method to deal with the sensitivity analysis of symmetric fuzzy linear programming problem $\left(\mathrm{P}_{1}\right)$.

In this section, an alternative method (named as Mehar method) is proposed for the same.

The steps of the proposed Mehar method are as follows.

Step 1. Use Step 1 to Step 3 of the Mehar method, proposed in Section 3 , to convert problem $\left(\mathrm{P}_{1}\right)$ into problem $\left(\mathrm{P}_{5}\right)$.

Step 2. Check which of the following cases is occurring.

Case $i$. If in the fuzzy linear programming problem $\left(\mathrm{P}_{1}\right)$ the fuzzy coefficient $\widetilde{c}_{j}$ of the $j$ th fuzzy variable $\tilde{x}_{j}$ is changed by $\widetilde{c}_{j}$, then replace the coefficient $c_{j}=\mathfrak{R}\left(\widetilde{c}_{j}\right)$ of the $j$ th variable $x_{j}$ of the crisp linear programming problem $\left(\mathrm{P}_{5}\right)$ by $c_{j}^{\prime}=\mathfrak{R}\left(\widetilde{c}_{j}^{\prime}\right)$. Go to Step 3.

Case ii. If the fuzzy coefficient $\widetilde{b}_{i}$ of the fuzzy linear programming problem $\left(\mathrm{P}_{1}\right)$ is changed by $\widetilde{b}_{i}^{\prime}$, then replace the coefficient $b_{i}=\Re\left(\widetilde{b}_{i}\right)$ of the crisp linear programming problem $\left(\mathrm{P}_{5}\right)$ by $b_{i}^{\prime}=\mathfrak{R}\left(\widetilde{b}_{i}^{\prime}\right)$. Go to Step 3 .

Case $i$ ii. If the coefficient $a_{i j}$ of the fuzzy linear programming problem $\left(\mathrm{P}_{1}\right)$ is changed by $a_{i j}^{\prime}$, then replace the coefficient $a_{i j}$ of the crisp linear programming problem $\left(\mathrm{P}_{5}\right)$ by $a_{i j}^{\prime}$. Go to Step 3.

Case iv. If a new symmetric trapezoidal fuzzy variable $\tilde{x}_{n+1}$ with coefficients $\vec{c}_{n+1}^{\prime}$ and $a_{i(n+1)}^{\prime}$ is added in the objective function and constraints, respectively, of the fuzzy linear programming problem $\left(\mathrm{P}_{1}\right)$, then add a new crisp variable $x_{n+1}$ with coefficients $c_{n+1}^{\prime}=\Re\left(\vec{c}_{n+1}^{\prime}\right)$ and $a_{i(n+1)}^{\prime}$ in the objective function and constraints, respectively, of the crisp linear programming problem $\left(\mathrm{P}_{5}\right)$. Go to Step 3 .

Case v. If a new fuzzy constraint $\sum_{j=1}^{n} a_{(m+1) j} \tilde{x}_{j} \approx \widetilde{b}_{m+1}$ is added in the fuzzy linear programming problem $\left(\mathrm{P}_{1}\right)$, then add a new constraint $\sum_{j=1}^{n} a_{(m+1) j} x_{j}=\mathfrak{R}\left(\widetilde{b}_{m+1}\right)$ in the crisp linear programming problem $\left(\mathrm{P}_{5}\right)$. Go to Step 3 .

Step 3. Use existing sensitivity analysis technique [12] to find the optimal solution of the modified crisp linear programming problem, obtained in Step 2, with the help of the optimal table of the crisp linear programming problem $\left(\mathrm{P}_{5}\right)$.

Step 4. Use Step 5 of the Mehar method, proposed in Section 3, to find the fuzzy optimal solution of the modified fuzzy linear programming problem with the help of the obtained optimal solution of the modified crisp linear programming problem. 


\section{Advantages of the Proposed Mehar Methods}

In this section, the advantages of the proposed Mehar methods over existing methods [2, 4-7, 9-11] are discussed.

(1) It is obvious from Step 5 of Mehar method, proposed in Section 3, that if the solution of problem $\left(\mathrm{P}_{5}\right)$ will exist, then infinite alternative solution of problem $\left(\mathrm{P}_{1}\right)$ will also exist. Hence, on applying the proposed Mehar methods, the drawbacks of existing methods $[2,4-7,9-11]$, discussed in Section 2, are resolved.

(2) Since, for applying the proposed Mehar methods, there is need to solve crisp linear programming problems, the existing and easily available software such as TORA and LINDO can be used for the same. However, for applying the existing methods $[2,4-7$, 9-11], there is need to solve fuzzy linear programming problems, so the existing and easily available software such as TORA and LINDO cannot be used and there is need to develop new software.

(3) To find the fuzzy optimal solution and to deal with the sensitivity analysis by using the existing methods $[2$, $4-7,9-11$ ], there is need to use arithmetic operations of fuzzy numbers. While if the proposed Mehar methods are used for the same, then there is need to use arithmetic operations of real numbers. Since it is much complicated to apply the arithmetic operations of fuzzy numbers as compared to the arithmetic operations of real numbers, so it is much easy to apply the proposed Mehar methods as compared to the existing methods $[2,4-7,9-11]$.

\section{Illustrative Examples}

In this section, the proposed Mehar methods are illustrated with the help of existing numerical problems.

Example 1 ([11, Example 1, pp. 178]). Solve the fuzzy linear programming problem

$$
\begin{aligned}
& \operatorname{maximize}\left[\tilde{z} \approx-(13,15,2,2) \tilde{x}_{1}\right. \\
& \left.-(12,14,3,3) \tilde{x}_{2}-(15,17,2,2) \tilde{x}_{3}\right] \\
& \text { subject to } 2 \tilde{x}_{1}+3 \tilde{x}_{2}+2 \tilde{x}_{3} \geq(45,55,6,6) \text {, } \\
& 4 \tilde{x}_{1}+3 \tilde{x}_{3} \succeq(60,80,8,8), \\
& 2 \tilde{x}_{1}+5 \tilde{x}_{2} \succeq(65,95,5,5), \\
& \tilde{x}_{1} \geq \tilde{0}, \quad \tilde{x}_{2} \geq \tilde{0}, \quad \tilde{x}_{3} \succeq \tilde{0} \text {. }
\end{aligned}
$$

Solution. Using the Mehar method, proposed in Section 3, the fuzzy optimal solution of the chosen problem can be obtained as follows.
Step 1. Using Step 1 of the proposed Mehar method, the chosen problem can be converted into problem $\left(\mathrm{P}_{6}\right)$ :

$$
\begin{gathered}
\operatorname{maximize}\left[\mathfrak{R}(\widetilde{z})=\Re\left(-(13,15,2,2) \tilde{x}_{1}-(12,14,3,3) \tilde{x}_{2}\right.\right. \\
\left.\left.-(15,17,2,2) \tilde{x}_{3}\right)\right]
\end{gathered}
$$

subject to $\Re\left(2 \tilde{x}_{1}+3 \tilde{x}_{2}+2 \tilde{x}_{3}\right) \geq \Re(45,55,6,6)$,

$$
\begin{aligned}
& \mathfrak{R}\left(4 \tilde{x}_{1}+3 \tilde{x}_{3}\right) \geq \mathfrak{R}(60,80,8,8), \\
& \mathfrak{R}\left(2 \tilde{x}_{1}+5 \tilde{x}_{2}\right) \geq \mathfrak{R}(65,95,5,5), \\
& \mathfrak{R}\left(\tilde{x}_{1}\right) \geq \mathfrak{R}(\widetilde{0}), \quad \mathfrak{R}\left(\tilde{x}_{2}\right) \geq \mathfrak{R}(\widetilde{0}), \\
& \mathfrak{R}\left(\tilde{x}_{3}\right) \geq \mathfrak{R}(\widetilde{0}) .
\end{aligned}
$$

Step 2. Using Step 2 of the proposed Mehar method, the problem $\left(\mathrm{P}_{6}\right)$ can be converted into problem $\left(\mathrm{P}_{7}\right)$ :

$$
\begin{gathered}
\operatorname{maximize}\left[\mathfrak{R}(\widetilde{z})=-\mathfrak{R}(13,15,2,2) \mathfrak{R}\left(\tilde{x}_{1}\right)\right. \\
-\mathfrak{R}(12,14,3,3) \mathfrak{R}\left(\tilde{x}_{2}\right) \\
\left.-\mathfrak{R}(15,17,2,2) \mathfrak{R}\left(\tilde{x}_{3}\right)\right]
\end{gathered}
$$

subject to $2 \mathfrak{R}\left(\tilde{x}_{1}\right)+3 \mathfrak{R}\left(\tilde{x}_{2}\right)+2 \mathfrak{R}\left(\tilde{x}_{3}\right) \geq \mathfrak{R}(45,55,6,6)$,

$$
\begin{aligned}
& 4 \Re\left(\tilde{x}_{1}\right)+3 \mathfrak{R}\left(\tilde{x}_{3}\right) \geq \mathfrak{R}(60,80,8,8), \\
& 2 \mathfrak{R}\left(\tilde{x}_{1}\right)+5 \mathfrak{R}\left(\tilde{x}_{2}\right) \geq \mathfrak{R}(65,95,5,5), \\
& \mathfrak{R}\left(\tilde{x}_{1}\right) \geq \mathfrak{R}(\widetilde{0}), \quad \mathfrak{R}\left(\tilde{x}_{2}\right) \geq \mathfrak{R}(\widetilde{0}), \\
& \mathfrak{R}\left(\tilde{x}_{3}\right) \geq \mathfrak{R}(\widetilde{0}) .
\end{aligned}
$$

Step 3. Using Step 3 of the proposed Mehar method, the problem $\left(\mathrm{P}_{7}\right)$ can be converted into problem $\left(\mathrm{P}_{8}\right)$ :

$$
\begin{aligned}
& \text { maximize }\left[z=-14 x_{1}-13 x_{2}-16 x_{3}\right] \\
& \text { subject to } 2 x_{1}+3 x_{2}+2 x_{3} \geq 50, \\
& 4 x_{1}+3 x_{3} \geq 70, \\
& 2 x_{1}+5 x_{2} \geq 80, \\
& x_{1} \geq 0, \quad x_{2} \geq 0, \quad x_{3} \geq 0 .
\end{aligned}
$$

Step 4. On solving the problem $\left(\mathrm{P}_{8}\right)$, the obtained optimal solution is

$$
x_{1}=\frac{35}{2}, \quad x_{2}=9, \quad x_{3}=0 .
$$

Step 5. Using Step 5 of the proposed Mehar method, all the symmetric trapezoidal fuzzy numbers $\tilde{x}_{1}, \tilde{x}_{2}$, and $\tilde{x}_{3}$ such that $\mathfrak{R}\left(\tilde{x}_{1}\right)=35 / 2, \mathfrak{R}\left(\tilde{x}_{2}\right)=9$, and $\mathfrak{R}\left(\tilde{x}_{3}\right)=0$ will be the fuzzy optimal solutions of the problem $\left(\mathrm{P}_{2}\right)$; for example, the following four are also fuzzy optimal solutions of the problem $\left(\mathrm{P}_{2}\right)$. 
(1) $\tilde{x}_{1}=(10,25,3,3), \tilde{x}_{2}=(8,10,2,2), \tilde{x}_{3}=(-1,1,3,3)$.

(2) $\tilde{x}_{1}=(17,18,4,4), \tilde{x}_{2}=(9,9,3,3), \tilde{x}_{3}=(-3,3,4,4)$.

(3) $\tilde{x}_{1}=(12,23,3,3), \tilde{x}_{2}=(6,12,4,4), \tilde{x}_{3}=(-2,2,3,3)$.

(4) $\tilde{x}_{1}=(16,19,2,2), \widetilde{x}_{2}=(7,11,8,8), \widetilde{x}_{3}=(-6,6,5,5)$.

Example 2 ([11, Example 2, pp. 179]). Solve the fuzzy linear programming problem $\left(\mathrm{P}_{9}\right)$ :

$\operatorname{Maximize}\left[\tilde{z} \approx(-1,-1,1,1) \tilde{x}_{1}+(-4,-4,4,4) \tilde{x}_{2}\right.$

$$
\left.+(-2,-1,3,3) \tilde{x}_{3}+(1,1,1,1) \tilde{x}_{4}\right]
$$

Subject to $\widetilde{x}_{1}-2 \widetilde{x}_{2}+\widetilde{x}_{3}-\tilde{x}_{4} \geq(-2,-2,3,3)$,

$$
\begin{aligned}
& 2 \widetilde{x}_{1}+\widetilde{x}_{2}+2 \widetilde{x}_{3}-2 \widetilde{x}_{4} \approx(2,3,1,1), \\
& \tilde{x}_{1}-3 \widetilde{x}_{3}+\widetilde{x}_{4} \geq(1,4,3,3), \\
& \tilde{x}_{1} \geq \widetilde{0}, \quad \widetilde{x}_{2} \geq \widetilde{0}, \quad \widetilde{x}_{3} \geq \widetilde{0}, \quad \widetilde{x}_{4} \geq \widetilde{0} .
\end{aligned}
$$

Solution. Using the Mehar method, proposed in Section 3, the fuzzy optimal solution of the problem $\left(\mathrm{P}_{9}\right)$ can be obtained as follows.

Step 1. Using Step 1 of the proposed Mehar method, the problem $\left(\mathrm{P}_{9}\right)$ can be converted into problem $\left(\mathrm{P}_{10}\right)$ :

$$
\begin{gathered}
\operatorname{maximize}\left[\mathfrak{R}(\widetilde{z})=\Re\left((-1,-1,1,1) \tilde{x}_{1}+(-4,-4,4,4) \tilde{x}_{2}\right.\right. \\
\left.\left.+(-2,-1,3,3) \widetilde{x}_{3}+(1,1,1,1) \widetilde{x}_{4}\right)\right]
\end{gathered}
$$

subject to $\Re\left(\tilde{x}_{1}-2 \widetilde{x}_{2}+\tilde{x}_{3}-\tilde{x}_{4}\right) \geq \mathfrak{R}(-2,-2,3,3)$,

$$
\begin{aligned}
& \mathfrak{R}\left(2 \widetilde{x}_{1}+\widetilde{x}_{2}+2 \widetilde{x}_{3}-2 \widetilde{x}_{4}\right)=\mathfrak{R}(2,3,1,1), \\
& \mathfrak{R}\left(\widetilde{x}_{1}-3 \widetilde{x}_{3}+\widetilde{x}_{4}\right) \geq \mathfrak{R}(1,4,3,3), \\
& \mathfrak{R}\left(\widetilde{x}_{1}\right) \geq \mathfrak{R}(\widetilde{0}), \quad \mathfrak{R}\left(\widetilde{x}_{2}\right) \geq \mathfrak{R}(\widetilde{0}), \\
& \mathfrak{R}\left(\widetilde{x}_{3}\right) \geq \mathfrak{R}(\widetilde{0}), \quad \mathfrak{R}\left(\widetilde{x}_{4}\right) \geq \mathfrak{R}(\widetilde{0}) .
\end{aligned}
$$

Step 2. Using Step 2 of the proposed Mehar method, the problem $\left(\mathrm{P}_{10}\right)$ can be converted into problem $\left(\mathrm{P}_{11}\right)$ :

$$
\begin{aligned}
\operatorname{maximize} & {\left[\Re(\widetilde{z})=\mathfrak{R}(-1,-1,1,1) \mathfrak{R}\left(\widetilde{x}_{1}\right)+\mathfrak{R}(-4,-4,4,4)\right.} \\
& \Re\left(\widetilde{x}_{2}\right)+\mathfrak{R}(-2,-1,3,3) \mathfrak{R}\left(\widetilde{x}_{3}\right) \\
& \left.+\mathfrak{R}(1,1,1,1) \mathfrak{R}\left(\tilde{x}_{4}\right)\right]
\end{aligned}
$$

subject to $\Re\left(\widetilde{x}_{1}\right)-2 \Re\left(\widetilde{x}_{2}\right)+\Re\left(\tilde{x}_{3}\right)-\mathfrak{R}\left(\widetilde{x}_{4}\right)$

$$
\begin{aligned}
& \geq \mathfrak{R}(-2,-2,3,3), \\
& 2 \mathfrak{R}\left(\widetilde{x}_{1}\right)+\mathfrak{R}\left(\widetilde{x}_{2}\right)+2 \mathfrak{R}\left(\widetilde{x}_{3}\right)-2 \mathfrak{R}\left(\widetilde{x}_{4}\right) \\
& =\mathfrak{R}(2,3,1,1), \\
& \mathfrak{R}\left(\widetilde{x}_{1}\right)-3 \mathfrak{R}\left(\tilde{x}_{3}\right)+\mathfrak{R}\left(\tilde{x}_{4}\right) \geq \mathfrak{R}(1,4,3,3), \\
& \mathfrak{R}\left(\widetilde{x}_{1}\right) \geq \mathfrak{R}(\widetilde{0}), \quad \mathfrak{R}\left(\widetilde{x}_{2}\right) \geq \mathfrak{R}(\widetilde{0}), \\
& \mathfrak{R}\left(\widetilde{x}_{3}\right) \geq \mathfrak{R}(\widetilde{0}), \quad \mathfrak{R}\left(\widetilde{x}_{4}\right) \geq \mathfrak{R}(\widetilde{0}) .
\end{aligned}
$$

Step 3. Using Step 3 of the proposed Mehar method, the problem $\left(\mathrm{P}_{11}\right)$ can be converted into problem $\left(\mathrm{P}_{12}\right)$ :

$$
\begin{aligned}
& \text { maximize }\left[z=-x_{1}-4 x_{2}-\frac{3}{2} x_{3}+x_{4}\right] \\
& \text { subject to } x_{1}-2 x_{2}+x_{3}-x_{4} \geq-2, \\
& 2 x_{1}+x_{2}+2 x_{3}-2 x_{4}=\frac{5}{2}, \\
& x_{1}-3 x_{3}+x_{4} \geq \frac{5}{2}, \\
& x_{1} \geq 0, \quad x_{2} \geq 0, \quad x_{3} \geq 0, \quad x_{4} \geq 0 .
\end{aligned}
$$

Step 4. On solving the problem $\left(\mathrm{P}_{12}\right)$, the obtained optimal solution is

$$
x_{1}=\frac{15}{8}, \quad x_{2}=0, \quad x_{3}=0, \quad x_{4}=\frac{5}{8} .
$$

Step 5. Using Step 5 of the proposed Mehar method, all the symmetric trapezoidal fuzzy numbers $\widetilde{x}_{1}, \widetilde{x}_{2}, \widetilde{x}_{3}$, and $\widetilde{x}_{4}$ such that $\mathfrak{R}\left(\tilde{x}_{1}\right)=15 / 8, \mathfrak{R}\left(\tilde{x}_{2}\right)=0, \mathfrak{R}\left(\tilde{x}_{3}\right)=0$, and $\Re\left(\widetilde{x}_{4}\right)=5 / 8$ will be the fuzzy optimal solutions of the problem $\left(\mathrm{P}_{9}\right)$; for example, the following four are also fuzzy optimal solutions of the problem $\left(\mathrm{P}_{9}\right)$.

(1) $\tilde{x}_{1}=(7 / 4,2,4,4), \tilde{x}_{2}=(-2,2,3,3), \widetilde{x}_{3}=(-1,1,3,3)$, $\tilde{x}_{4}=(1 / 4,1,3 / 2,3 / 2)$.

(2) $\tilde{x}_{1}=(3 / 2,9 / 4,2,2), \widetilde{x}_{2}=(-1,1,1 / 2,1 / 2), \widetilde{x}_{3}=$ $(-5,5,2,2), \tilde{x}_{4}=(1 / 2,3 / 4,9,9)$.

(3) $\tilde{x}_{1}=(3 / 4,3,3,3), \widetilde{x}_{2}=(0,0,6,6), \widetilde{x}_{3}=(-2,2,4,4)$, $\tilde{x}_{4}=(7 / 12,2 / 3,1,1)$.

(4) $\tilde{x}_{1}=(1 / 2,13 / 4,4,4), \widetilde{x}_{2}=(-3,3,2,2), \widetilde{x}_{3}=$ $(0,0,2,2), \widetilde{x}_{4}=(5 / 8,5 / 8,5,5)$. 
Example 3 ([11, Example 3, pp. 181]). Find range of $\lambda$ so that there is no change in the fuzzy optimal solution of the fuzzy linear programming problem $\left(\mathrm{P}_{9}\right)$ on replacing $\widetilde{c}_{2}=$ $(-4,-4,4,4)$ by $\widetilde{c}_{2}^{\prime}=\widetilde{c}_{2}+(3,5,6,6) \lambda$.

Solution. Using the Mehar method, proposed in Section 4, the desired range for $\lambda$ can be obtained as follows.

Step 1. Since $\widetilde{c}_{2}=(-4,-4,4,4)$ is replaced by $\widetilde{c}_{2}^{\prime}=\widetilde{c}_{2}+$ $\lambda(3,5,6,6)$, using Step 2 of the proposed Mehar method replacing $\mathfrak{R}\left(\widetilde{c}_{2}\right)=\mathfrak{R}(-4,-4,4,4)=-4$ by $\mathfrak{R}\left(\widetilde{c}_{2}^{\prime}\right)=$ $\mathfrak{R}(-4,-4,4,4)+\mathfrak{R}(3,5,6,6) \lambda=-4+4 \lambda$ the crisp linear programming problem $\left(\mathrm{P}_{12}\right)$ can be written as

$$
\begin{aligned}
& \text { maximize }(z)=-x_{1}+(-4+4 \lambda) x_{2}-\frac{3}{2} x_{3}+x_{4} \\
& \text { subject to } x_{1}-2 x_{2}+x_{3}-x_{4} \geq-2, \\
& \qquad x_{1}+x_{2}+2 x_{3}-2 x_{4}=\frac{5}{2}, \\
& \qquad x_{1}-3 x_{3}+x_{4} \geq \frac{5}{2}, \\
& \qquad x_{1} \geq 0, \quad x_{2} \geq 0, \quad x_{3} \geq 0, \quad x_{4} \geq 0 .
\end{aligned}
$$

Step 2. Using existing sensitivity analysis technique [12] it can be easily found that if $\lambda \leq 7 / 8$, then the optimal solution of the modified crisp linear programming problem and hence the fuzzy optimal solution of the modified fuzzy linear programming problem will remain unchanged.

Example 4 ([11, Example 4, pp. 183]). Find range of $\lambda$ so that there is no change in the fuzzy optimal solution of the fuzzy linear programming problem $\left(\mathrm{P}_{9}\right)$ on replacing $\widetilde{b}_{1}=$ $(-2,-2,3,3), \widetilde{b}_{2}=(2,3,1,1)$, and $\widetilde{b}_{3}=(1,4,3,3)$ by $\widetilde{b}_{1}^{\prime}=$ $\widetilde{b}_{1}+(4,2,1,1) \lambda, \widetilde{b}_{2}^{\prime}=\widetilde{b}_{2}+(3,2,5,5) \lambda$, and $\widetilde{b}_{3}^{\prime}=\widetilde{b}_{3}+(1,2,3,3) \lambda$, respectively.

Solution. Using the Mehar method, proposed in Section 4, the desired range for $\lambda$ can be obtained as follows.

Step 1. Since $\widetilde{b}_{1}=(-2,-2,3,3), \widetilde{b}_{2}=(2,3,1,1)$, and $\widetilde{b}_{3}=$ $(1,4,3,3)$ are replaced by $\tilde{b}_{1}^{\prime}=\widetilde{b}_{1}+(4,2,1,1) \lambda, \tilde{b}_{2}^{\prime}=\widetilde{b}_{2}+$ $(3,2,5,5) \lambda$, and $\widetilde{b}_{3}^{\prime}=\widetilde{b}_{3}+(1,2,3,3) \lambda$, using Step 2 of the proposed Mehar method replacing $\mathfrak{R}\left(\widetilde{b}_{1}\right)=\mathfrak{R}(-2,-2,3,3)=$ $-2, \mathfrak{R}\left(\widetilde{b}_{2}\right)=\mathfrak{R}(2,3,1,1)=5 / 2$, and $\mathfrak{R}\left(\widetilde{b}_{3}\right)=\mathfrak{R}(1,4,3,3)=$ $5 / 2$ by $\mathfrak{R}\left(\widetilde{b}_{1}^{\prime}\right)=\mathfrak{R}\left(\widetilde{b}_{1}\right)+\mathfrak{R}(4,2,1,1) \lambda=-2+3 \lambda, \mathfrak{R}\left(\widetilde{b}_{2}^{\prime}\right)=$ $\mathfrak{R}\left(\tilde{b}_{2}\right)+\mathfrak{R}(3,2,5,5) \lambda=(5 / 2)+(5 / 2) \lambda$, and $\mathfrak{R}\left(\tilde{b}_{3}^{\prime}\right)=\mathfrak{R}\left(\tilde{b}_{3}\right)+$ $\mathfrak{R}(1,2,3,3) \lambda=(5 / 2)+(3 / 2) \lambda$ the crisp linear programming problem $\left(\mathrm{P}_{12}\right)$ can be written as

$$
\begin{aligned}
& \operatorname{maximize}(z)=-x_{1}-4 x_{2}-\frac{3}{2} x_{3}+x_{4} \\
& \text { subject to } x_{1}-2 x_{2}+x_{3}-x_{4} \geq-2+3 \lambda, \\
& 2 x_{1}+x_{2}+2 x_{3}-2 x_{4}=\frac{5}{2}+\frac{5}{2} \lambda,
\end{aligned}
$$

$$
\begin{aligned}
& x_{1}-3 x_{3}+x_{4} \geq \frac{5}{2}+\frac{3}{2} \lambda, \\
& x_{1} \geq 0, \quad x_{2} \geq 0, \quad x_{3} \geq 0, \quad x_{4} \geq 0 .
\end{aligned}
$$

Step 2. Using existing sensitivity analysis technique [12], it can be easily found that if $-15 / 11 \leq \lambda \leq 13 / 7$, then the optimal solution of the modified crisp linear programming problem and hence the fuzzy optimal solution of the modified fuzzy linear programming problem will remain unchanged.

Example 5 ([11, Example 5, pp. 184]). Find range of $\lambda$ so that there is no change in the fuzzy optimal solution of the fuzzy linear programming problem $\left(\mathrm{P}_{9}\right)$ on replacing the coefficients $-2,1,0$ and $1,2,-3$ of the fuzzy variables $\tilde{x}_{2}$ and $\tilde{x}_{3}$ of the first, second, and third constraints by $-2+\lambda, 1+2 \lambda,-\lambda$ and $1-3 \lambda, 2+2 \lambda,-3+2 \lambda$, respectively.

Solution. Using the Mehar method, proposed in Section 4, the desired range for $\lambda$ can be obtained as follows.

Step 1. Since the coefficients $-2,1,0$ and 1, 2, -3 of the fuzzy variables $\tilde{x}_{2}$ and $\tilde{x}_{3}$ of the first, second, and third constraints are replaced by $-2+\lambda, 1+2 \lambda,-\lambda$ and $1-3 \lambda, 2+2 \lambda,-3+2 \lambda$, respectively, using Step 2 of the proposed Mehar method, replacing the coefficients $-2,1,0$ and $1,2,-3$ of the fuzzy variables $\tilde{x}_{2}$ and $\tilde{x}_{3}$ of the first, second, and third constraints by $-2+\lambda, 1+2 \lambda,-\lambda$ and $1-3 \lambda, 2+2 \lambda,-3+2 \lambda$, respectively, the crisp linear programming problem $\left(\mathrm{P}_{12}\right)$ can be written as

$$
\begin{aligned}
& \text { maximize }(z)=-x_{1}-4 x_{2}-\frac{3}{2} x_{3}+x_{4} \\
& \text { subject to } x_{1}+(-2+\lambda) x_{2}+(1-3 \lambda) x_{3}-x_{4} \geq-2, \\
& 2 x_{1}+(1+2 \lambda) x_{2}+(2+2 \lambda) x_{3}-2 x_{4}=\frac{5}{2}, \\
& x_{1}+(-\lambda) x_{2}+(-3+2 \lambda) x_{3}+x_{4} \geq \frac{5}{2}, \\
& x_{1} \geq 0, \quad x_{2} \geq 0, \quad x_{3} \geq 0, \quad x_{4} \geq 0 .
\end{aligned}
$$

Step 2. Using existing sensitivity analysis technique [12], it can be easily found that if $\lambda \leq 1 / 2$, then the optimal solution of the modified crisp linear programming problem and hence the fuzzy optimal solution of the modified fuzzy linear programming problem will remain unchanged.

Example 6 ([11, Example 6, pp. 187]). Find range of $\lambda$ so that there is no change in the fuzzy optimal solution of the fuzzy linear programming problem $\left(\mathrm{P}_{9}\right)$ on replacing the coefficients 1,2 , and 1 of the fuzzy variables $\tilde{x}_{1}$ of the first, second, and third constraints by $1+2 \lambda, 2$, and $1-\lambda$, respectively.

Solution. Using the Mehar method, proposed in Section 4, the desired range for $\lambda$ can be obtained as follows.

Step 1 . Since the coefficients 1,2 , and 1 of the fuzzy variables $\tilde{x}_{1}$ of the first, second, and third constraints are replaced by 
$1+2 \lambda, 2$, and $1-\lambda$ respectively, using Step 2 of the proposed Mehar method, replacing the coefficients 1,2 , and 1 of the fuzzy variables $\tilde{x}_{1}$ of the first, second, and third constraints by $1+2 \lambda, 2$, and $1-\lambda$, respectively, the crisp linear programming problem $\left(\mathrm{P}_{12}\right)$ can be written as

$$
\begin{aligned}
& \text { maximize }(z)=-x_{1}-4 x_{2}-\frac{3}{2} x_{3}+x_{4} \\
& \text { subject to }(1+2 \lambda) x_{1}-2 x_{2}+x_{3}-x_{4} \geq-2, \\
& 2 x_{1}+x_{2}+2 x_{3}-2 x_{4}=\frac{5}{2}, \\
& (1-\lambda) x_{1}-3 x_{3}+x_{4} \geq \frac{5}{2}, \\
& x_{1} \geq 0, \quad x_{2} \geq 0, \quad x_{3} \geq 0, \quad x_{4} \geq 0 .
\end{aligned}
$$

Step 2. Using existing sensitivity analysis technique [12] it can be easily found that if $1 \leq \lambda \leq 2$, then the optimal solution of the modified crisp linear programming problem and hence the fuzzy optimal solution of the modified fuzzy linear programming problem will remain unchanged.

Example 7 ([11, Example 7, pp. 188]). Find the fuzzy optimal solution of the fuzzy linear programming problem $\left(\mathrm{P}_{9}\right)$ obtained by adding the fuzzy constraint $\tilde{x}_{1}-3 \tilde{x}_{2}+2 \tilde{x}_{4} \preceq$ $(-1,3,4,4)$ in the existing fuzzy linear programming problem [11, Example 2, pp. 179].

Solution. Using the Mehar method, proposed in Section 4, the fuzzy optimal solution of the modified fuzzy linear programming problem can be obtained as follows.

Step 1 . Since the fuzzy constraint $\tilde{x}_{1}-3 \tilde{x}_{2}+2 \tilde{x}_{4} \preceq(-1,3,4,4)$ is added in the fuzzy linear programming problem, using Step 2 of the Mehar method adding the $\mathfrak{R}\left(\widetilde{x}_{1}-3 \tilde{x}_{2}+2 \widetilde{x}_{4}\right) \leq$ $\mathfrak{R}(-1,3,4,4)$, that is, $x_{1}-3 x_{2}+2 x_{4} \leq 1$, the crisp linear programming problem $\left(\mathrm{P}_{12}\right)$ can be written as

$$
\begin{aligned}
& \text { maximize }(z)=-x_{1}-4 x_{2}-\frac{3}{2} x_{3}+x_{4} \\
& \text { subject to } x_{1}-2 x_{2}+x_{3}-x_{4} \geq-2, \\
& 2 x_{1}+x_{2}+2 x_{3}-2 x_{4}=\frac{5}{2}, \\
& x_{1}-3 x_{3}+x_{4} \geq \frac{5}{2}, \\
& x_{1}-3 x_{2}+2 x_{4} \leq 1, \\
& x_{1} \geq 0, \quad x_{2} \geq 0, \quad x_{3} \geq 0, \quad x_{4} \geq 0 .
\end{aligned}
$$

Step 2. Using existing sensitivity analysis technique [12], optimal solution of modified crisp linear programming problem is

$$
x_{1}=\frac{37}{22}, \quad x_{2}=\frac{17}{22}, \quad x_{3}=0, \quad x_{4}=\frac{18}{22} .
$$

Step 3. Using Step 5 of the proposed Mehar method, all the symmetric trapezoidal fuzzy numbers $\tilde{x}_{1}, \tilde{x}_{2}, \tilde{x}_{3}$, and $\tilde{x}_{4}$ such that $\mathfrak{R}\left(\tilde{x}_{1}\right)=37 / 22, \mathfrak{R}\left(\tilde{x}_{2}\right)=17 / 22, \mathfrak{R}\left(\tilde{x}_{3}\right)=0$, and $\mathfrak{R}\left(\tilde{x}_{4}\right)=$ $18 / 22$ will be the fuzzy optimal solutions of the chosen fuzzy linear programming problem.

\section{Conclusion}

On the basis of the present study, it can be concluded that all the fuzzy linear programming problems which can be solved by the existing methods $[2,4-7,9-11]$ can also be solved by the proposed Mehar methods. However, it is much easy to apply the proposed Mehar methods as compared to the existing methods [2, 4-7, 9-11]. Also on applying the proposed Mehar methods the drawbacks of the existing methods [2, 4-7, 911] are resolved. Hence, it is better to use the proposed Mehar methods as compared to the existing methods [2, 4-7, 9-11].

The proposed Mehar method is applicable only for fuzzy linear programming problems with symmetric trapezoidal fuzzy numbers. In future, proposing new methods for solving fuzzy linear programming problems with nonsymmetric trapezoidal fuzzy numbers may be tried.

\section{Appendix}

In this section, some basic definitions are presented [11].

Definition 8. A fuzzy number on $R$ (real line) is said to be a symmetric trapezoidal fuzzy number, if there exist real numbers $a^{L}$ and $a^{U}, a^{L} \leq a^{U}$ and $\alpha>0$, such that

$$
\mu_{\widetilde{a}}(x)= \begin{cases}\frac{x}{\alpha}+\frac{\alpha-a^{L}}{\alpha}, & x \in\left[a^{L}-\alpha, a^{L}\right], \\ 1, & x \in\left[a^{L}, a^{U}\right], \\ -\frac{x}{\alpha}+\frac{a^{U}+\alpha}{\alpha}, & x \in\left[a^{U}, a^{U}+\alpha\right], \\ 0, & \text { otherwise. }\end{cases}
$$

A symmetric trapezoidal fuzzy number $\tilde{a}$ is denoted by $\tilde{a}=$ $\left(a^{L}, a^{U}, \alpha, \alpha\right)$.

Let $\tilde{a}=\left(a^{L}, a^{U}, \alpha, \alpha\right)$ and $\tilde{b}=\left(b^{L}, b^{U}, \beta, \beta\right)$ be two symmetric trapezoidal fuzzy numbers. The arithmetical operations on $\tilde{a}$ and $\widetilde{b}$ are as follows.

(1) $x>0, x \in R ; x \tilde{a}=\left(x a^{L}, x a^{U}, x \alpha, x \alpha\right)$.

(2) $x<0, x \in R ; x \widetilde{a}=\left(x a^{U}, x a^{L},-x \alpha,-x \alpha\right)$.

(3) $\tilde{a}+\tilde{b}=\left(a^{L}+b^{L}, a^{U}+b^{U}, \alpha+\beta, \alpha+\beta\right)$.

(4) $\tilde{a} \otimes_{G} \tilde{b}=\left(\left(\left(a^{L}+a^{U}\right) / 2\right)\left(\left(b^{L}+b^{U}\right) / 2\right)-w,\left(\left(a^{L}+\right.\right.\right.$ $\left.\left.\left.a^{U}\right) / 2\right)\left(\left(b^{L}+b^{U}\right) / 2\right)+w,\left|a^{U} \beta+b^{U} \alpha\right|,\left|a^{U} \beta+b^{U} \alpha\right|\right)$.

where $w=(h-k) / 2, k=\min \left(a^{L} b^{L}, a^{L} b^{U}, a^{U} b^{L}, a^{U} b^{U}\right)$, and $h=\max \left(a^{L} b^{L}, a^{L} b^{U}, a^{U} b^{L}, a^{U} b^{U}\right)$.

Definition 9. Let $\widetilde{a}=\left(a^{L}, a^{U}, \alpha, \alpha\right)$ and $\widetilde{b}=\left(b^{L}, b^{U}, \beta, \beta\right)$ be two symmetric trapezoidal fuzzy numbers.

Define the relation as

$\widetilde{a} \preceq \widetilde{b}$ if and only if either $\mathfrak{R}(\widetilde{a})<\Re(\widetilde{b})$ (in this case, we can also write $\widetilde{a} \prec \widetilde{b}$ )

or $\mathfrak{R}(\widetilde{a})=\mathfrak{R}(\widetilde{b}), b^{L}<a^{L}$, and $a^{U}=b^{U}$ 


$$
\begin{aligned}
& \text { or } \mathfrak{R}(\widetilde{a})=\mathfrak{R}(\widetilde{b}), b^{L}=a^{L} \text {, and } a^{U}=b^{U} \text { and } \alpha \leq \beta, \\
& \text { where } \Re(\widetilde{a})=\left(a^{L}+a^{U}\right) / 2 \text { and } \Re(\tilde{b})=\left(b^{L}+b^{U}\right) / 2 .
\end{aligned}
$$

(In the last two cases, we can also write $\widetilde{a} \approx \widetilde{b}$ and say that $\widetilde{a}$ and $\tilde{b}$ are equivalent.)

Remark 10. Two symmetric trapezoidal fuzzy numbers $\left(a^{L}, a^{U}, \alpha, \alpha\right)$ and $\left(b^{L}, b^{U}, \beta, \beta\right)$ are equivalent if and only if $\mathfrak{R}(\widetilde{a})=\mathfrak{R}(\widetilde{b})$.

In this case, we simply write $\left(a^{L}, a^{U}, \alpha, \alpha\right) \approx\left(b^{L}, b^{U}, \beta, \beta\right)$ and it is to be noted that $a^{L}$ need not be equal to $b^{L}$ or $a^{U}$ need not be equal to $b^{U}$, but $\left(a^{L}, a^{U}, \alpha, \alpha\right)-\left(b^{L}, b^{U}, \beta, \beta\right) \approx$ $(-h, h, \alpha+\beta, \alpha+\beta)$, where $h=\left(b^{U}-a^{L}\right) \geq 0$.

\section{Conflict of Interests}

The authors declare that there is no conflict of interests regarding the publication of this paper.

\section{Acknowledgments}

The authors would like to thank the anonymous referees for various suggestions which have led to an improvement in both the quality and clarity of the paper. Dr. Amit Kumar would like to acknowledge the adolescent blessings of Mehar (lovely daughter of his cousin sister Dr. Parmpreet Kaur). He believes that Mata Vaishno Devi has appeared on the earth in the form of Mehar and without Mehar's blessings it was not possible to think of the ideas presented in this paper.

\section{References}

[1] L. A. Zadeh, "Fuzzy sets," Information and Control, vol. 8, pp. 338-353, 1965.

[2] K. Ganesan and P. Veeramani, "Fuzzy linear programs with trapezoidal fuzzy numbers," Annals of Operations Research, vol. 143, no. 1, pp. 305-315, 2006.

[3] S. H. Nasseri and N. Mahdavi-Amiri, "Some duality results on linear programming problems with symmetric fuzzy numbers," Fuzzy Information and Engineering, vol. 1, pp. 59-66, 2009.

[4] S. H. Nasseri, A. Ebrahimnejad, and S. Mizuno, "Duality in fuzzy linear programming with symmetric trapezoidal numbers," Applications and Applied Mathematics, vol. 5, no. 10, pp. 1469-1484, 2010.

[5] A. Ebrahimnejad, S. H. Nasseri, and F. H. Lotfi, "Bounded linear programs with trapezoidal fuzzy numbers," International Journal of Uncertainty, Fuzziness and Knowledge-Based Systems, vol. 18, no. 3, pp. 269-286, 2010.

[6] A. Ebrahimnejad, "A primal-dual simplex algorithm for solving linear programming problems with symmetric trapezoidal fuzzy numbers," Applied Mathematics, vol. 2, no. 6, pp. 676-684, 2011.

[7] A. Ebrahimnejad and S. H. Nasseri, "Linear programmes with trapezoidal fuzzy numbers: a duality approach," International Journal of Operational Research, vol. 13, no. 1, pp. 67-89, 2012.

[8] A. Kumar and J. Kaur, "A new method for solving fuzzy linear programs with trapezoidal fuzzy numbers," Journal of Fuzzy Set Valued Analysis, vol. 2011, pp. 1-12, 2011.
[9] A. Ebrahimnejad, "Some new results in linear programs with trapezoidal fuzzy numbers: finite convergence of the Ganesan and Veeramani's method and a fuzzy revised simplex method," Applied Mathematical Modelling, vol. 35, no. 9, pp. 4526-4540, 2011.

[10] A. Ebrahimnejad and J. L. Verdegay, "A novel approach for sensitivity analysis in linear programs withtrapezoidal fuzzy numbers," Journal of Intelligent and Fuzzy Systems. In press.

[11] B. Kheirfam and J.-L. Verdegay, "The dual simplex method and sensitivity analysis for fuzzy linear programming with symmetric trapezoidal numbers," Fuzzy Optimization and Decision Making, vol. 12, no. 2, pp. 171-189, 2013.

[12] H. A. Taha, Operations Research: An Introduction, Prentice Hall, Upper Saddle River, NJ, USA, 2003. 


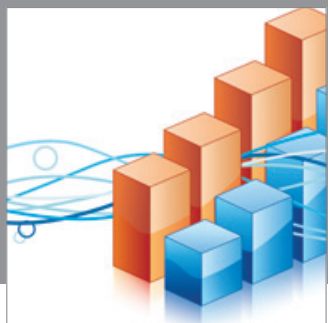

Advances in

Operations Research

mansans

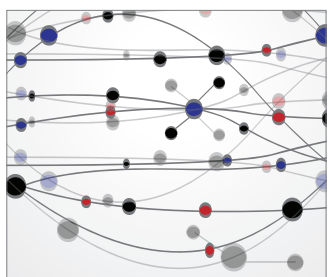

The Scientific World Journal
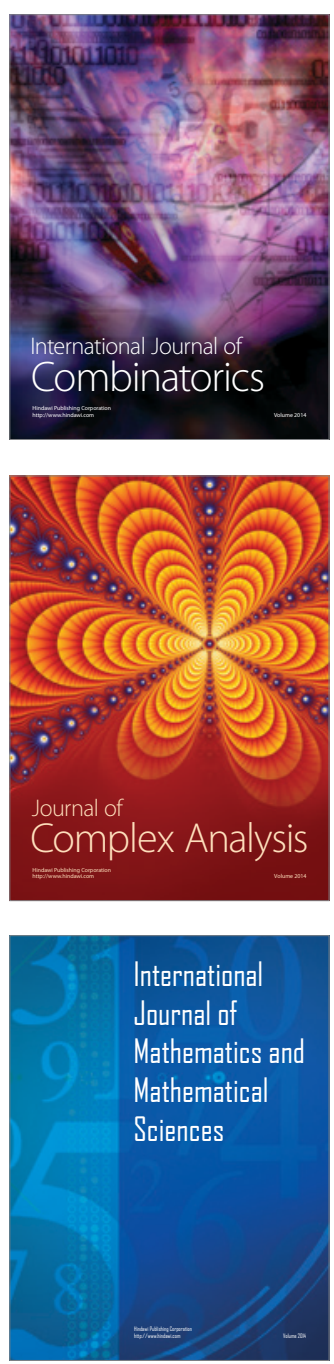
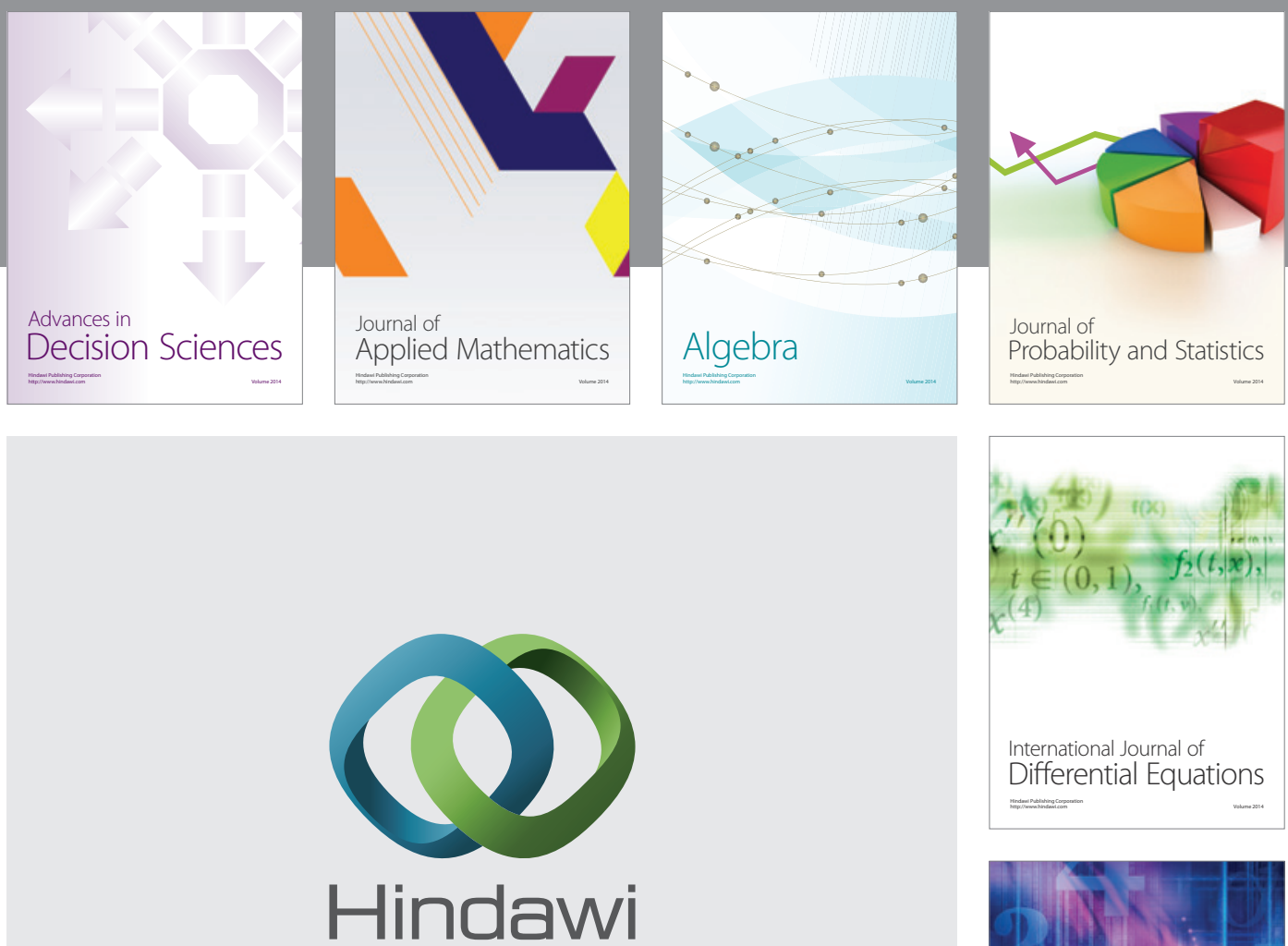

Submit your manuscripts at http://www.hindawi.com
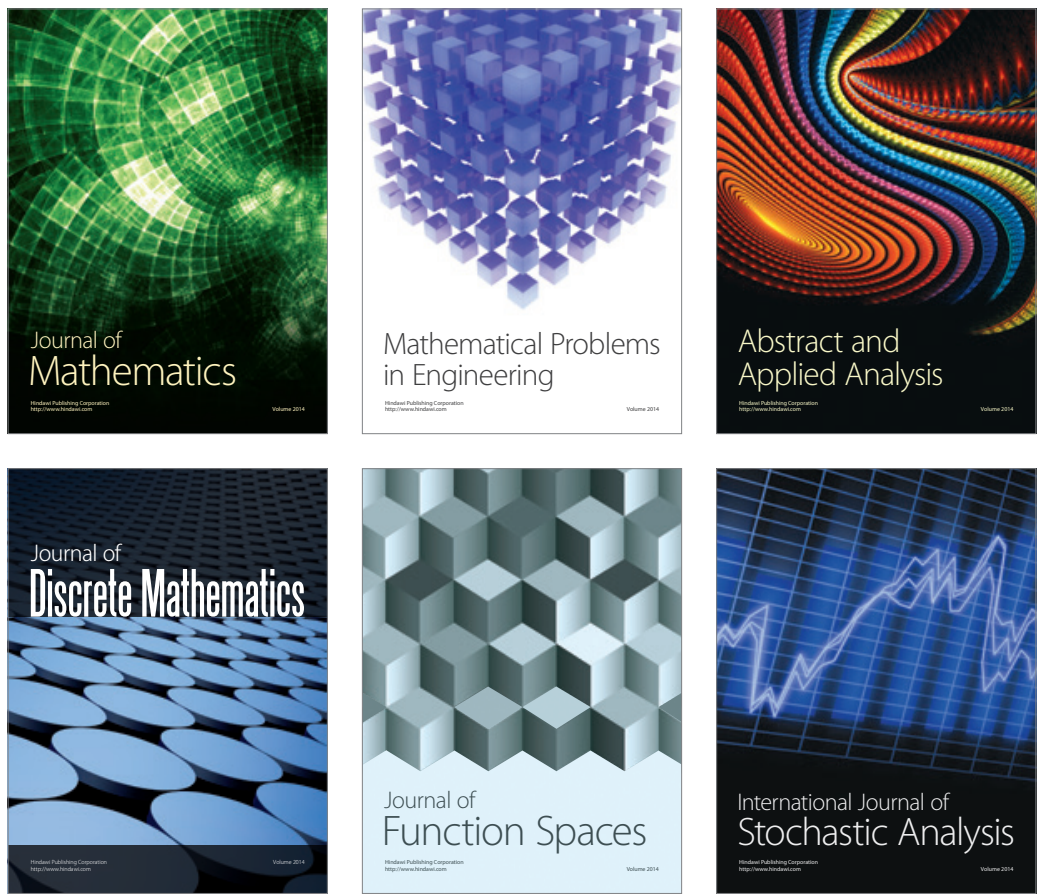

Journal of

Function Spaces

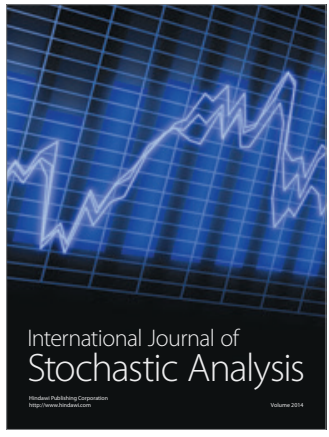

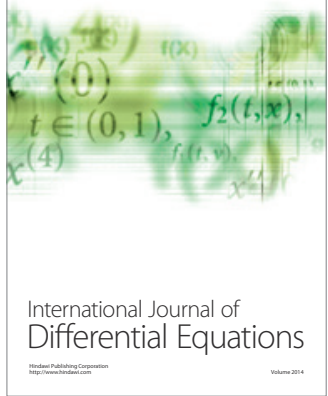
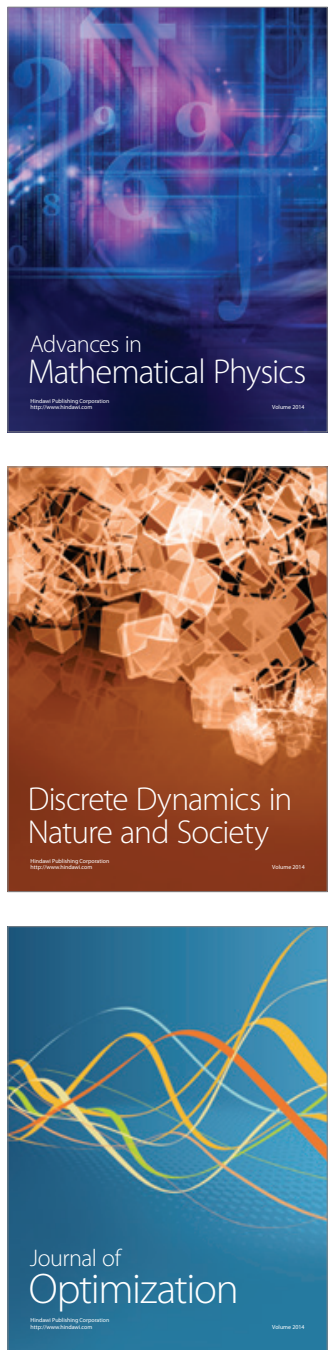